\title{
Ultrasonic Regeneration of Granular Activated Carbon
}

\author{
Guojun Zhang, ${ }^{1, *}$ Sanfan Wang, ${ }^{2}$ and Zhongzhou Liu ${ }^{1}$ \\ ${ }^{1}$ Research Center for Eco-Environmental Sciences \\ Chinese Academy of Sciences \\ Beijing, 100085, People's Republic of China \\ ${ }^{2}$ Department of Environmental Engineering \\ Lanzhou Railway University \\ Lanzhou, 730070, People's Republic of China
}

\begin{abstract}
A new ultrasonic method for regeneration of granular activated carbon was developed. Experimental results showed that the method was effective. The operation time and type of ultrasound, the kind of adsorbate, and the size of granular carbon were investigated in the process of ultrasonic regeneration. The ultrasonic regeneration has many advantages, such as lower energy consumption, simpler process equipment, lower carbon loss, higher recovery of valuable substances, etc.
\end{abstract}

Key words: ultrasound; granular activated carbon; regeneration

\section{INTRODUCTION}

G RANULAR ACTIVATED CARBOn (GAC) is widely used in a number of ways such as deodorization and removal of residual chlorine, pigments, pesticides, detergents, aromatic, and other heterocyclic organic compounds (Chiang et al., 1997). Although GAC is very effective in these application processes, the unit cost is very high. It is concluded that the regeneration of the exhausted activated carbon on-site is the most economical and applicable method for lowering the operation cost (Cheremisinoff, 1978). Regeneration methods, including thermal treatment, chemical treatment, biological treatment, electrochemical treatment, and microwave treatment methods, are still being investigated and developed (Dobrevski and Zvezdova, 1989; Fang and Lai, 1996; Chiang et al., 1997; Zhang et al., 1999). Among these methods, the thermal regeneration method is currently the most promising technique for regenerating the GAC (Suzuki et al., 1978; Urano et al., 1982). However, in the thermal regeneration process, high temperature up to the vaporization point is needed to heat activated carbon, adsorbates, and water. Thus, a large amount of energy is consumed, and the regeneration equipment is complicated. In fact, if sufficient energy is applied, the adsorbates will be separated from adsorbent surface and enter bulk solution, and a process for regenerating activated carbon can be achieved. To avoid excessive use of energy, the ultrasonic regeneration method was proposed (Bao et al, 1995; Wang, 1998). However, no evidence in the literature suggests any research on using ultrasound to regenerate activated carbon has been completed. This article presents preliminary data through our ongoing study in this respect.

*Corresponding author: Research Center for Eco-Environmental Sciences, Chinese Academy of Science, P.O. Box 2871, Beijing, 10085, People's Republic of China. Phone: 86-10-62849195; Fax: 86-10-62923441;E-mail: zhangguojunmh@yahoo.com 
Ultrasonic waves have frequencies over $16 \mathrm{kHz}$, and spread in the form of a spherical wave. When a wave contacts different phase surfaces or forms another ultrasonic crest with a certain angle, huge compression forces will be produced on the surface or in the wave colliding area. During wave reflection, many tiny "cavitation bubbles" will be formed. The sizes of "cavitation bubbles" are up to several tens of a nanometer, and they have lives of only hundredths of a second or much shorter. When "cavitation bubbles" burst, the local temperature can reach thousands of degrees, and the local pressure can reach up to hundreds of bars (Mason and Lorimer, 1988). When pressure and temperature act on the adsorbent surface, energy is delivered to the adsorbates, followed by the acceleration of the thermal motion of the adsorbates. The adsorbates will separate from the adsorbent surface and enter the bulk solution. In addition, ultrasonic wave has strong blending functions. In the transmission process, ultrasonic wave stirs the medium with thousands of pulses per minute. Moreover, when "cavitation bubbles" burst, the pulse causes the substances, which are separated from the adsorbent surface, to enter the bulk solution quickly; subsequently, the whole system reaches equilibrium (Wang, 1998). It is remarkable that ultrasonic waves act only locally, and do not need to heat a large volume of water and activated carbon. Consequently, this method consumes less energy.

In this work, ultrasonic regeneration was performed, and effects of operation time and type, the kind of adsorbates, carbon size, ultrasonic frequency, and output power on desorption ratio were investigated and discussed. The preliminary data are provided. It was demonstrated that the ultrasonic regeneration was efficient and economical. This novel regeneration method has good potential.

\section{EXPERIMENTAL}

\section{Experimental apparatuses}

In this work, both static and dynamic regeneration experiments were carried out. Schematic diagrams of the apparatuses used for the static and dynamic experimental studies are shown in Fig. 1(a) and (b), respectively.

The ultrasonic reactor was equipped with a piezoelectric ceramic transducer. A round stainless steel trough was used. The inner dimension was $12.5 \mathrm{~cm}$, and the height was $8 \mathrm{~cm}$. The transducer was glued to the outer bottom of the trough, which produced ultrasonic waves at $33 \mathrm{kHz}, 50 \mathrm{~W}$.

\section{Preparation of samples}

The experiments were performed to evaluate the effectiveness of ultrasonic wave in regenerating GAC-containing phenol, alkyl benzene sulfonate (ABS), and $\mathrm{Cr}(\mathrm{VI})$. The following procedure was used to prepare the samples of exhausted activated carbon.

Phenol, ABS, and $\mathrm{Cr}(\mathrm{VI})$ as adsorbates were used to represent low molecular organic matter, a general organic molecule, and a heavy metal ion, respectively. The corresponding concentration was 3,5 , and $1 \mathrm{mg} / \mathrm{L}$. GAC was packed in a column. The solutions were filtered through the column with a flow rate of $10 \mathrm{~m} / \mathrm{h}$. Except for the carbon size effect experiments, the particle size of GAC was $0.3-0.4 \mathrm{~mm}$. The GAC layer was $90 \mathrm{~cm}$ high and

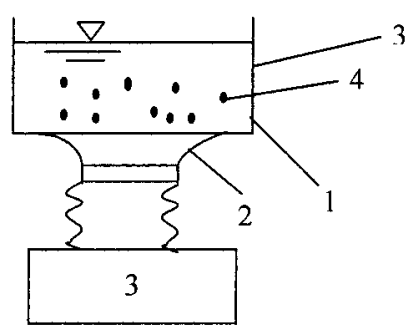

(a)

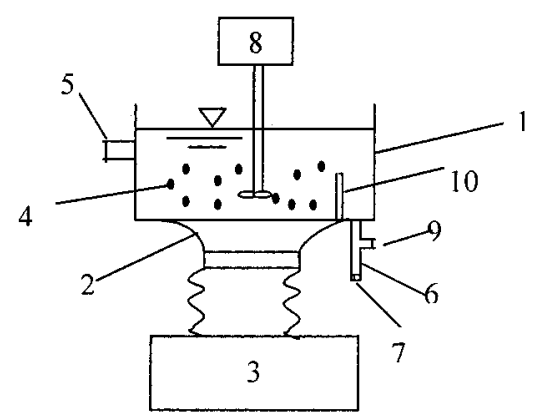

(b)

Figure 1. Schematic diagrams of the apparati. (a) Static experimental apparatus: 1. regeneration vessel; 2. piezoelectric ceramics transducer; 3. controller; 4. activated carbon. (b) Dynamic experimental apparatus: 1. regeneration vessel; 2. piezoelectric ceramics transducer; 3 . controller; 4. activated carbon; 5. inlet of feed; 6 . outlet of regeneration wastewater; 7.100 mesh nylon net; 8. Stirrer; 9. outlet of activated carbon; 10. baffle. 
the water temperature was $14-15^{\circ} \mathrm{C}$. When the concentration of the adsorbate in the outlet water was $50 \%$ of that in the inlet water, the adsorption was considered complete. At this moment, $7.1 \mathrm{mg} / \mathrm{mL}$ phenol, 56.1 $\mathrm{mg} / \mathrm{mL}$ ABS, $11.7 \mathrm{mg} / \mathrm{mL} \mathrm{Cr}(\mathrm{VI})$ were adsorbed, respectively. All of the GAC was taken out from the column. Then the upper and lower layer of activated carbon were mixed homogeneously and stored in wet conditions.

\section{Calculations of the adsorbate in carbon}

The dynamic adsorption experiments were carried out before regeneration. Every $30 \mathrm{~min}$, the adsorbate concentration in outlet solution was determined and compared with the initial value. The volume of outlet solution was also recorded in this period. The following equation was developed to calculate the amount of adsorbate in activated carbon before regeneration:

$$
Q=\left(C_{0} V_{0}-\Sigma C_{y} V_{h}\right) / m
$$

where $C_{0} V_{0}$ is the initial mass of adsorbate in the solution and $\Sigma C_{h} V_{h}$ is the residual mass of adsorbate in the initial solution; $\mathrm{C}_{0}$ is the initial concentration of adsorbate; $V_{0}$ is the initial volume of solution; $C_{h}$ is the average concentration of outlet solution every $30 \mathrm{~min}$; $V_{h}$ is the volume of outlet solution in the $30 \mathrm{~min}$; and $m$ is the mass of activated carbon.

In addition, the adsorbate on activated carbon after regeneration can be calculated according to the following equation:

$$
R=Q-\left(C_{r} V_{r}\right) / m
$$

where $Q$ is the adsorbate on activated carbon before regeneration according to the calculation from Equation (1); $C_{r}$ is the average concentration of regeneration wastewater; and $V_{r}$ is the total volume of regeneration wastewater.

\section{Experimental methods}

Static regeneration experiment. Water and the wet GAC, which was previously saturated in the dynamic adsorption experiment, were charged into the vessel. The volume ratio of carbon to water was 1:9. After ultrasonic regeneration, the regeneration wastewater was separated from composite solution by a tube wrapped with 100 mesh nylon net. The amounts of adsorbates in the regeneration solutions and in activated carbon were analyzed and calculated before and after regeneration, respectively.

Dynamic regeneration experiment. The regeneration vessel was equipped with a stirrer. The mixture of water and carbon was pumped to the regeneration vessel with a centrifugal feed pump at a given flow rate. The mixture continuously flowed from the vessel through a baffle, and the carbon was separated from water immediately by a separator wrapped with 100 mesh nylon net. Finally, the separated solution and the adsorbates in activated carbon were analyzed.

\section{Analytical methods}

The concentrations of phenol, ABS, and Cr(VI) were determined according to the procedures in Standard Methods (APHA et al., 1980). The concentration of phenol was quantified by a direct photometric method, through the absorbance measurement (at $510 \mathrm{~nm}$ ) of a colored antipyrine dye in an aqueous solution that was formed by the reaction between the steam-distillable phenols and 4-aminoantipyrine at a $\mathrm{pH}$ of $10.0 \pm 0.2$ in the presence of potassium ferricyanide. ABS was measured by methylene blue-active substances method, and $\mathrm{Cr}(\mathrm{VI})$ was analyzed by a colorimetric method.

\section{RESULTS AND DISCUSSION}

\section{Effect of operation time and operation style on desorption ratio}

Theoretical analysis and experimental results show that ultrasonic waves are effective for the regeneration of activated carbon. In the ultrasonic regeneration of GAC adsorbing ABS, we observed that foam was produced. As shown in Fig. 2(a), (b), and (c), and $30-40 \%$ of adsorbates were stripped. The regeneration ratio increases with operation time. At a certain operation time, new equilibrium is achieved on the surface where "cavitation" occurs more easily. At this operation time, the quantity of substances stripped from the interior of activated carbon clearly decreases. Therefore, desorption ratio does not increase with longer operation time.

Figure 2 shows that the dynamic regeneration provides higher desorption than static regeneration. Because the adsorbates can be drained in time during the dynamic regeneration, the adsorption-desorption equilibrium can move towards the description direction; this leads to a more thorough desorption process. The above-mentioned processes are in accordance with the mass action law.

\section{Effect of different adsorbates on desorption ratio}

Desorption ratios of different adsorbates are different. Figure 3(a) and (b) shows that desorption ratios with a large absolute adsorption quantity are higher than those with small absolute adsorption quantity. The main reason is that different adsorbates can form different multiple layers through physical adsorption. The higher 
(a)

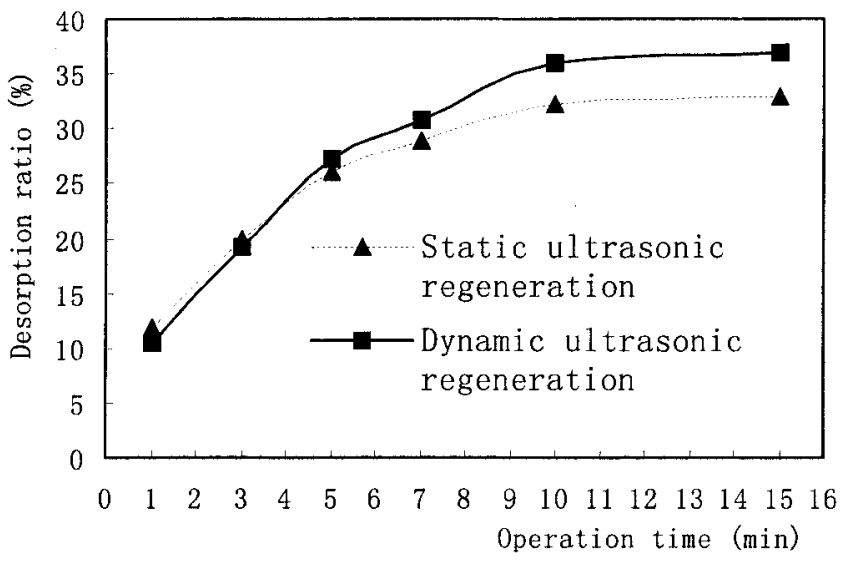

(b)

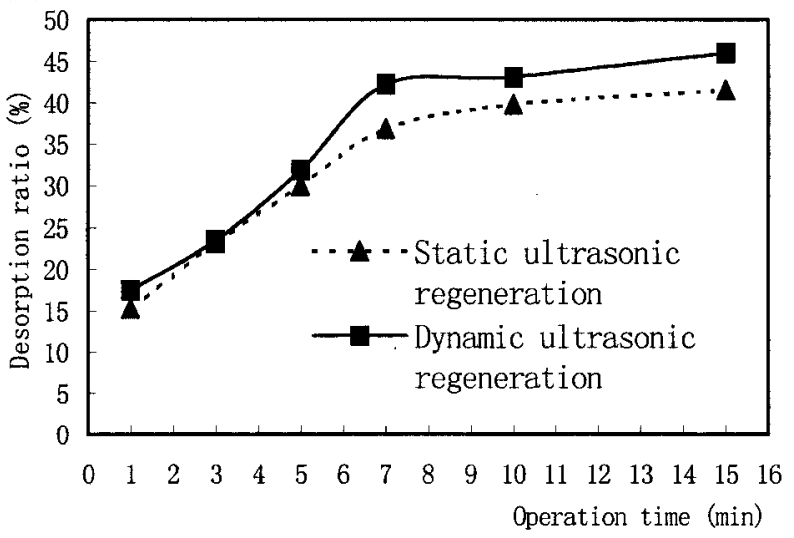

(c)

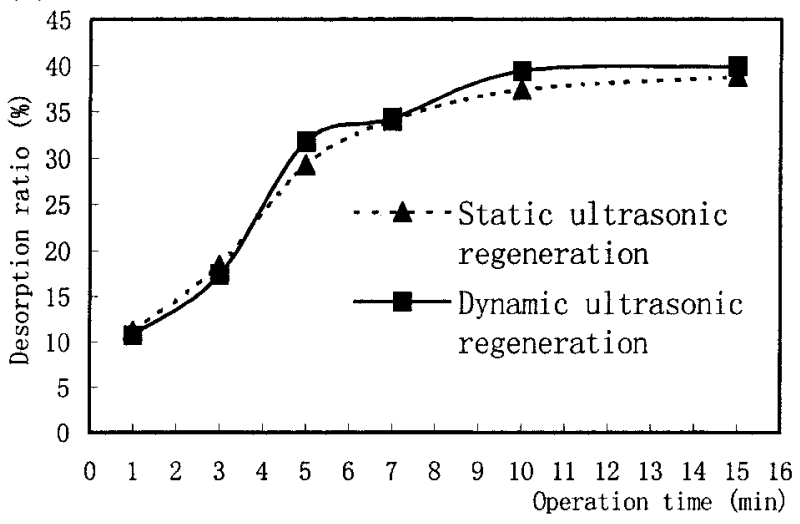

Figure 2. Effects of operation time and operation type on desorption ratio. (a) Effects of operation time and operation type on phenol desorption ratio. (b) Effects of operation time and operation style on ABS desorption ratio. (c) Effects of operation time and operation type on $\mathrm{Cr}(\mathrm{VI})$ desorption ratio. The initial regeneration water temperature was $14^{\circ} \mathrm{C}$, and the final regeneration water temperature was $16.5^{\circ} \mathrm{C}$. the adsorption quantity, the thicker the adsorption layer. This leads to a weaker adsorption interaction at the outer layer and easier desorption. When the adsorption between some substances and activated carbon belong to reversible chemical adsorption (hydrogen bond or chemical bond) or electrostatic adsorption (electric charge attraction), the adsorption force is stronger and desorption is more difficult. In addition, desorption of big molecules is easier than small molecules. Experiments and theoretical analysis show that not all adsorbates in activated carbon can be regenerated by ultrasonic waves. Desorption ratios of different adsorbates by ultrasonic regeneration are different. If the GAC, which adsorbs various substances, is regenerated by ultrasonic waves, some kinds of substances can be accumulated in the carbon. In addition, unlike chemical and thermal regeneration, ultrasonic regeneration cannot change the structure and the form of adsorbates; therefore, it has the advantages of regenerating the GAC, which is applied to concentrate, gather, and recover some valuable substances.

\section{Effect of carbon size on desorption ratio}

The particle size of GAC has an important effect on desorption ratio. It is difficult for ultrasonic waves to reach the interior of GAC with cavities smaller than 10 $\mathrm{nm}$. "Cavitation" function mainly occurs on the surface or in some large cavities. After adsorbates on the surface are stripped, the interior adsorbates gradually move to the carbon surface by pore diffusion. It is then moved into solution. However, because pore diffusion is slower, it is impossible for the interior of activated carbon to be regenerated completely. It is obvious that some substances still exist in the interior of GAC and cannot be stripped by ultrasound. Our experiments with GAC using ABS for six continuous periods show that after ultrasonic regeneration, the adsorption capacity changes greatly only in the first period. However, in the five successive adsorption-desorption-adsorption periods, the adsorption capacity of GAC changes minimally after regeneration. In the first adsorption-desorption-adsorption period, adsorption occurs both on the surface and in the interior of GAC, but desorption only occurs on the surface and in some large cavities. Therefore, adsorption capacity can only recover about $40 \%$ of the original capacity. Both the adsorption and desorption effects focus mainly on the surface and in some large cavities, so the capacity of GAC changes slightly in the five successive periods.

It is found that ultrasonic regeneration acts mainly on the surface of small particles of activated carbon. As shown in the Fig. 4(a), (b), and (c), a smaller carbon size is related directly to a higher ultrasonic regeneration ra- 
tio. When the size reduces to a critical value, it is difficult to separate carbon particle from the regeneration wastewater quickly. In addition, the regeneration of small-sized carbon particles is limited by other factors such as water flow resistance and recoil loss.

\section{Effect of ultrasonic frequency and output power on desorption ratio}

Due to the limitation of experimental measurements, the measurement and test of varying ultrasonic frequency and output power were not conducted. However, our analysis suggests that the adsorption heat of different adsorbates is different, and the bond forces between different adsorbates and GAC are different. If different fre- quency ultrasonic waves are used to regenerate different adsorbates, the best operation frequency and operation time will depend on the adsorbates. Therefore, additional experiments are needed to determine the best regeneration parameters.

\section{Advantage of ultrasonic regeneration over thermal method}

Table 1 shows the operation conditions of ultrasonic regeneration and thermal method. Table 2 shows the regeneration results under these conditions. Compared with the thermal method, the ultrasonic regeneration is superior in respect of regeneration time, procedure, and temperature.

(a)

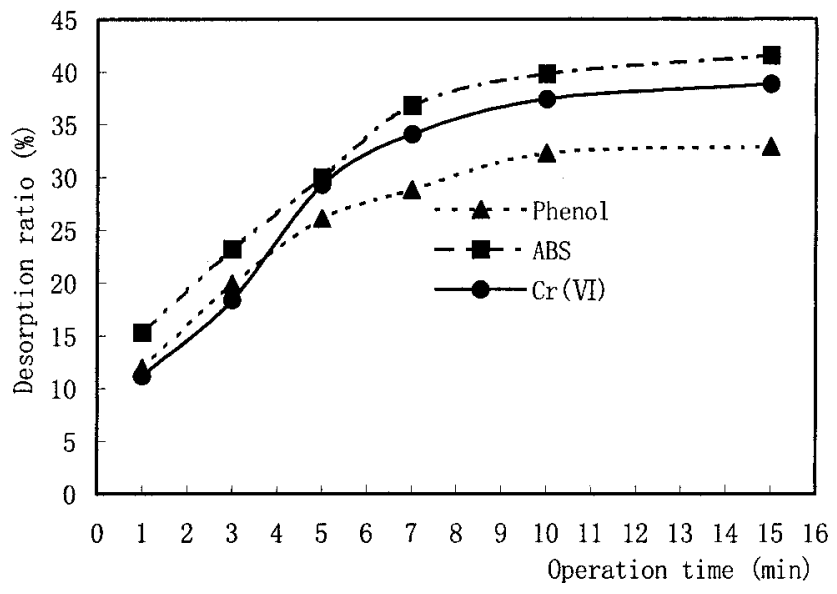

(b)

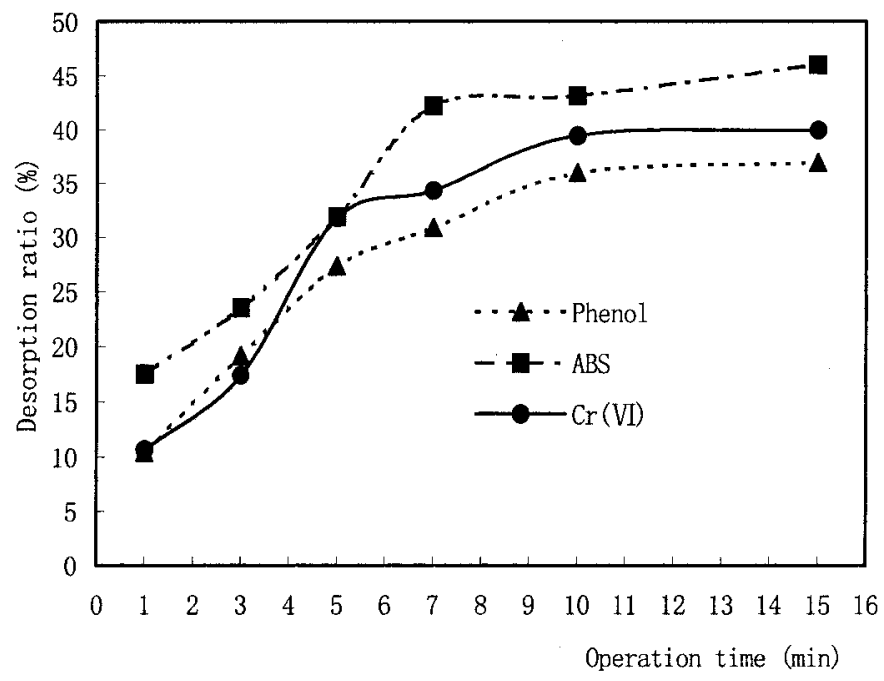

Figure 3. Effect of different adsorbates on desorption ratio in ultrasonic regeneration. (a) Static ultrasonic regeneration. (b) Dynamic ultrasonic regeneration. The initial regeneration water temperature was $14^{\circ} \mathrm{C}$, and the finial regeneration water temperature was $16.5^{\circ} \mathrm{C}$. The initial concentrations of phenol, ABS, and $\mathrm{Cr}(\mathrm{VI})$ were $7.1 \mathrm{mg} / \mathrm{L}, 56.1 \mathrm{mg} / \mathrm{L}$, and $11.7 \mathrm{mg} / \mathrm{L}, \mathrm{respectively}$. 
(a)

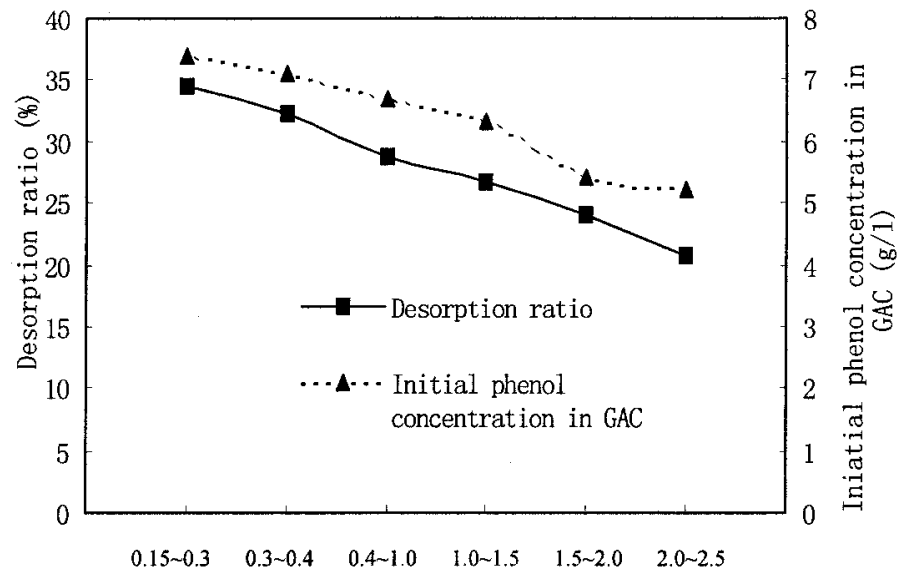

Carbon particle size (mm)

(b)

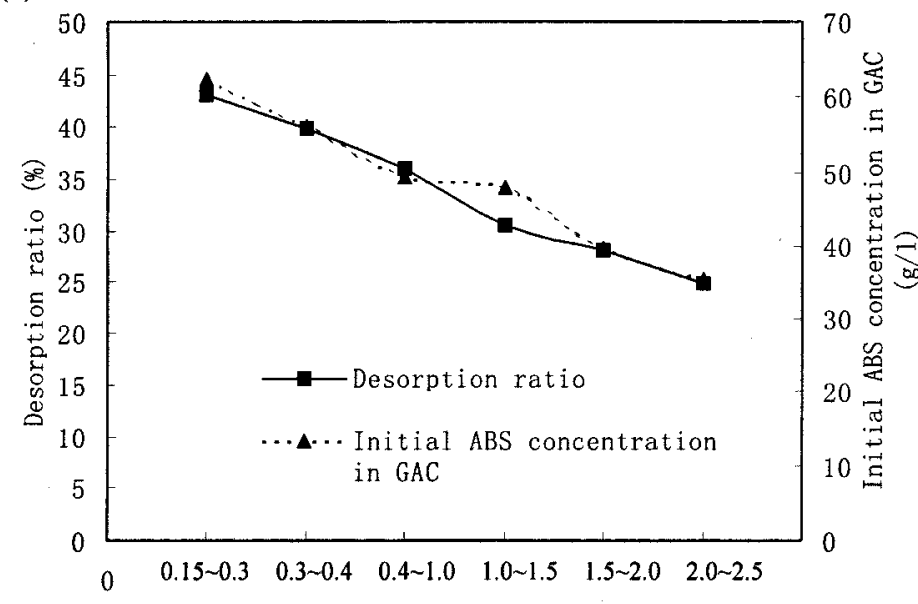

Carbon particle size $(\mathrm{mm})$

(c)

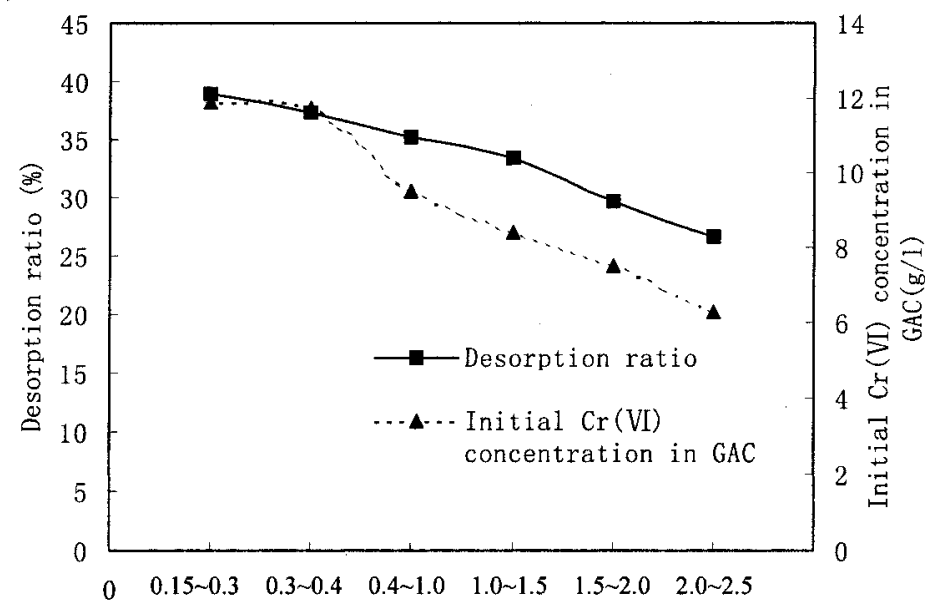

Figure 4. Effect of GAC particle size on desorption ratio in ultrasonic regeneration. (a) Effect of GAC particle size on phenol desorption ratio in static ultrasonic regeneration. (b) Effect of GAC particle size on ABS desorption ratio in static ultrasonic regeneration. (c) Effect of GAC particle size on $\mathrm{Cr}(\mathrm{VI})$ desorption ratio in static ultrasonic regeneration. The initial regeneration water temperature was $14^{\circ} \mathrm{C}$, and the finial regeneration water temperature was $16.5^{\circ} \mathrm{C}$. Ultrasonic operation time was $10 \mathrm{~min}$. 
Table 1. Operating conditions of ultrasonic regeneration and thermal method.

\begin{tabular}{|c|c|c|c|}
\hline \multicolumn{2}{|c|}{ Thermal regeneration } & \multicolumn{2}{|c|}{ Ultrasonic regeneration } \\
\hline Process & Time (min) & Process & Time (min) \\
\hline Centrifugalization & 10 & Feed & 0.5 \\
\hline Preheating & 20 & & \\
\hline Regeneration & 30 & Regeneration & 10 \\
\hline Cooling & 20 & & \\
\hline Elution & 10 & & \\
\hline Outlet & 2 & Outlet & 0.5 \\
\hline Total & 92 & Total & 11 \\
\hline
\end{tabular}

$0.3 \sim 0.4 \mathrm{~mm} \mathrm{GAC}$ absorbing $\mathrm{ABS}$ and $33 \mathrm{kHz}, 50 \mathrm{~W}$ ultrasonic wave were used in these parallel experiments.

In traditional thermal regeneration, the loss of GAC, which achieves $5-8 \%$, is a persistent problem. Carbon loss in ultrasonic regeneration is less than that in thermal regeneration. Many tiny carbon particles can be observed in the regeneration process, and making the regeneration wastewater slightly black. After separation from the regeneration wastewater, carbon particles are dried, weighed, and compared with the initial drying activated carbon. The loss of activated carbon, which is regenerated for $10 \mathrm{~min}$, is less than $0.8 \%$.

The most important characteristic of ultrasonic regeneration is that energy consumption is small. The energy efficiency of ultrasonic regeneration is high. In the regeneration process, the carbon particles, which are dispersed into solution, provide large phase surfaces. Therefore, ultrasonic "cavitation" function focuses mainly on carbon particles. The solution temperature increases only by $2-3^{\circ} \mathrm{C}$ after regeneration, showing that a large amount of energy is not dissipated as heat. When most energy transforms into effective work, adsorbates can acquire sufficient energy. Therefore, thermal motion of adsorbates is accelerated, allowing the adsorbates to leave easily from the adsorption surface, preventing readsorption. For $0.3-0.4 \mathrm{~mm}$ activated carbon particles, $0.08 \mathrm{kWh}$ is needed to achieve $40 \%$ desorption ratio in the regeneration of $85 \mathrm{~mL}$ activated carbon. If the power factor is 0.85 , the regeneration of $1 \mathrm{~m}^{3}$ wet activated carbon consumes $115 \mathrm{kWh}$.

In addition, ultrasonic regeneration has other advantages such as a simple regeneration process, low equip- ment cost, minimal carbon loss, low water consumption, and economic potential.

\section{CONCLUSIONS}

We proved the effective regeneration of GAC by ultrasonic waves. The main factors related with desorption ratio, such as operation time, operation type, different adsorbates, and carbon particle size, were investigated. For a certain adsorbate, a new equilibrium can be achieved on the carbon surface by prolonging the operation time. Dynamic regeneration is better than static regeneration due to more thorough desorption. In ultrasonic regeneration, the desorption ratio of different adsorbates is different because of the different forces between GAC and adsorbates. In addition, desorption ratio in ultrasonic regeneration depends on the size of carbon particles. Ultrasonic "cavitation" function mainly occurs on the surface or in some large cavities. The adsorption capacity changes greatly in the first regeneration period and slightly after two periods. Theoretical analyses show that other factors including ultrasonic output frequency and power influence the ultrasonic regeneration process.

The ultrasonic regeneration has many advantages, such as lower energy consumption, simpler process equipment, minimal carbon loss, and higher recovery of valuable substances. In conclusion, ultrasonic regeneration has good potential for providing more efficient use of energy and simpler regeneration procedure. However, ad-

Table 2. Desorption ratio changes of ultrasonic regeneration and thermal regeneration with operating time under the conditions in Table 1.

\begin{tabular}{llccc}
\hline Ultrasonic regeneration & Regeneration time (min) & 2 & 5 & 10 \\
& Desorption ratio (\%) & 19.4 & 31.1 & 42.9 \\
Thermal regeneration & Regeneration time (min) & 5 & 10 & 45.8 \\
& Desorption ratio (\%) & 11.7 & 23.9 & 30 \\
\hline
\end{tabular}


ditional experiments are still needed to determine the best regeneration parameters.

\section{ACKNOWLEDGMENT}

We would like to thank Dr. Jiantuan Ge and Dr. Xuefei Sun for the preparation of this manuscript.

\section{REFERENCES}

APHA, AWWA, and WPCF. (1980). Standard Methods for the Examination of Water and Wastewater, 14th ed. New York: APHA/AWWA/WPCF.

BAO, C., ZHAO, Y., FENG, R., and ZHU, C. (1995). Recovery of active charcoal from waste catalyst via ultrasonic wave. $J$. Nanjing Univ. (Nat. Sci. Ed.) 31(2), 242 (in Chinese).

CHEREMISINOFF, P.N. (1978). Carbon Adsorption Handbook, Ann Arbor, MI: Science Publishers Inc.

CHIANG, P.C., CHANG, E.E., and WU, J.S. (1997). Comparison of chemical and thermal regeneration of aromatic compounds on exhausted activated carbon. Water Sci. Technol. 35(7), 279-285.
DOBREVSKI, I., and ZVEZDOVA, L. (1989). Biological regeneration of activated carbon. Water Sci. Technol. 21(2), $141-143$.

FANG, C.S., and LAI, P.M.C. (1996). Microwave regeneration of spent powder activated carbon. Chem. Eng. Commun. 147, $17-27$.

MASON, T.J., and LORIMER, J.P. (1988). Sonochemistry: Theory, Applications and Uses of Ultrasound in Chemistry. Chichester, UK: Ellis Horwood.

SUZUKI, M., MISIC, D. KOYAMA, O., and KAWAZOL, K. (1978). Study of thermal regeneration of spent activated carbon. Chem. Eng. Sci. 33, 271.

URANO, K., OMORL, S., and YAMAMOTO, E. (1982). Prediction method for adsorption capacities of commercial activated carbon in removal of organic vapors. Environ. Sci. Technol. 16(1), 10.

WANG, S. (1998). Tentative researches on regeneration of activated carbon using ultrasonic method. Chin. Water Wastewater 14(2), 24 (in Chinese).

ZHANG, H., ZHONG, H., and YE, L. (1999). Regeneration of activated carbon by electrochemical method. Chem. Eng. 27(2), 31-33 (in Chinese). 